\title{
Estimating the Leverage Parameter of Continuous-time Stochastic Volatility Models Using High Frequency S\&P 500 and VIX*
}

\author{
Isao Ishida \\ Center for the Study of Finance and Insurance \\ Osaka University, Japan \\ Michael McAleer \\ Econometric Institute \\ Erasmus School of Economics \\ Erasmus University Rotterdam \\ The Netherlands \\ and \\ Tinbergen Institute, The Netherlands \\ and \\ Institute of Economic Research \\ Kyoto University, Japan \\ and \\ Department of Quantitative Economics \\ Complutense University of Madrid \\ Kosuke Oya \\ Graduate School of Economics \\ and \\ Center for the Study of Finance and Insurance \\ Osaka University, Japan
}

Revised: May 2011

* The authors are most grateful to two referees for helpful comments and suggestions. The first author wishes to thank Yusho Kaguraoka, Toshiaki Watanabe, and participants at the 2010 Annual Meeting of the Nippon Finance Association, the CSFI Nakanoshima Workshop 2009, and the Hiroshima University of Economics Financial Econometrics Workshop 2010 for valuable comments, and the Japan Society for the Promotion of Science (Grants-in-Aid for Scientific Research No. 20530265) for financial support. The second author is most grateful for the financial support of the Australian Research Council, National Science Council, Taiwan, and the Japan Society for the Promotion of Science. The third author is thankful for Grants-in-Aid for Scientific Research No. 22243021 from the Japan Society for the Promotion of Science. 


\begin{abstract}
This paper proposes a new method for estimating continuous-time stochastic volatility (SV) models for the S\&P 500 stock index process using intraday high-frequency observations of both the S\&P 500 index and the Chicago Board of Exchange (CBOE) implied (or expected) volatility index (VIX). Intraday high-frequency observations data have become readily available for an increasing number of financial assets and their derivatives in recent years, but it is well known that attempts to directly apply popular continuous-time models to short intraday time intervals, and estimate the parameters using such data, can lead to nonsensical estimates due to severe intraday seasonality. A primary purpose of the paper is to provide a framework for using intraday high frequency data of both the index estimate, in particular, for improving the estimation accuracy of the leverage parameter, $\rho$, that is, the correlation between the two Brownian motions driving the diffusive components of the price process and its spot variance process, respectively. As a special case, we focus on Heston's (1993) square-root SV model, and propose the realized leverage estimator for $\rho$, noting that, under this model without measurement errors, the "realized leverage," or the realized covariation of the price and VIX processes divided by the product of the realized volatilities of the two processes, is in-fill consistent for $\rho$. Finite sample simulation results show that the proposed estimator delivers more accurate estimates of the leverage parameter than do existing methods.
\end{abstract}

Keywords: Continuous time, high frequency data, stochastic volatility, S\&P 500, implied volatility, VIX.

JEL Classifications: G13, G17, G32. 


\section{Introduction}

The negative correlation between return and its volatility is one of the most salient empirical features of time series of equity price observations. Many variants of the continuous-time and discrete-time stochastic volatility (SV) and GARCH-type volatility models incorporating this feature in the dynamic equation for volatility have been proposed in the literature. This correlation in the underlying asset price or index affects the theoretical prices of options in such a way as to fit and explain partially the empirically observed "skew" patterns in the Black-Scholes options implied volatilities plotted against the strike prices ${ }^{1}$. Thus, such a correlation has attracted substantial attention in the asset pricing and financial econometrics literature.

Statistical estimation of this correlation for a particular type of continuous-time SV models is a primary focus of this paper. The negative price-volatility correlation is customarily referred to as "leverage" after Black's (1976) explanation based on the increased debt-equity ratio of a firm following its share price decrease raising its share price volatility ${ }^{2}$. In this paper, we also use the term "leverage" interchangeably with correlation between return and its volatility, without restriction regarding its sign.

For the analysis of derivatives, one-factor mean-reverting diffusion processes often augmented by jump components are commonly used as continuous-time models of the spot variance, among which the affine-drift square-root SV model of Heston (1993) enjoys popularity due to its analytical tractability. One-factor SV diffusion models incorporate leverage by allowing the two Brownian motions driving the price process and its volatility process, respectively, to be correlated. Even if the chosen parametric model is correctly specified, it requires an accurate estimate of this correlation, $\rho$, or the "leverage" parameter, together with the other parameters, for the model to be useful in derivatives pricing and hedging.

\footnotetext{
1 See, for example, Das and Sundram (1999).

2 For economic explanations in the equity case, see Bollerslev et al. (2006) and the references cited therein. The leverage concept does not apply to non-equity cases.
} 
In this paper, we propose a new method for estimating this leverage parameter, either individually or jointly with the other parameters, for a class of continuous-time SV models using high frequency intraday observations of the price and its "model-free" options implied volatility. Essentially, we propose to use the "realized leverage," or the realized correlation between the price and the model-free implied variance, for improving statistical inference ${ }^{3}$. The realized correlation between two series is the realized covariation divided by the product of the two realized volatilities. Although many papers, including Barndorff-Nielsen and Shephard (2004) and Vortelinos (2010), have studied the realized covariation and correlation between a pair of asset return series, the use of these quantities to measure leverage and to estimate the parameters of specific SV models, to the best of our knowledge, is novel.

We focus on the Heston SV model in this paper for two reasons. First, it is one of the most commonly used and important models among SV models. Hence, it is more than an example for merely illustrating the benefit of using high-frequency implied volatility data. Second, when it is correctly specified and there are no measurement errors, the realized leverage converges to $\rho$ in probability as the time intervals between observations shrink to zero, even if the length of the whole sample period is fixed. This property, together with the availability of analytical expressions for the conditional moments of realized variance, makes the Heston SV model a simple and clear example for showing the benefits of using the high frequency implied volatility data jointly with the S\&P 500 index data. Although $\rho$ cannot be backed out in this way for models other than the Heston SV model, using high frequency observations of both indices is likely to produce superior parameter estimates. Under the affine-drift constant-elasticity-of-variance (CEV) SV process, the realized leverage is not consistent except under the Heston SV special case, but appears to be robust as an estimator of $\rho$. For more exact inference for non-Heston models, simulated moments of realized measures, including the realized covariation or correlation, may be used.

\footnotetext{
${ }^{3}$ For estimating models other than the Heston SV model, it may be more convenient to use the moments of the realized covariation rather than the realized correlation.
} 
Intraday high frequency data have become readily available for an increasing number of financial assets and their derivatives in recent years. However, it is well known that attempts to apply directly popular continuous-time models that are intended to approximate financial processes at daily or lower frequencies to short intraday intervals of one hour or less, and estimate the parameters using high-frequency returns, say five-minute returns, lead to nonsensical parameter estimates due to intraday seasonality ${ }^{4}$ and various microstructure effects. For directly modeling returns at short time intervals, simple jump diffusion models are clearly not an accurate approximation, so that it is necessary to use fundamentally different approaches, such as the one pursued by Rydberg and Shephard (2003). However, such approaches, while important in empirically understanding microstructure phenomena, do not easily lend themselves to the analysis of derivatives.

While most of the time series estimators for simple continuous-time SV models proposed in the literature are implemented using daily data, due not only to the limitation of data availability but also to the above consideration, some authors have sought to extract information contained in high frequency intraday data for parameter estimation and jump identification, retaining simple jump diffusion models. Notable among them is Bollerslev and Zhou (2002), who proposed a GMM estimator for the Heston model and its several extensions using moment conditions based on conditional moments of the daily realized variance, which is a daily aggregate of short intraday squared returns. For estimating $\rho$, the BZ estimator relies on the cross moment of the daily closing price and the daily realized variance. The results of our finite sample simulation experiments using the Heston model indicate that their GMM estimator for $\rho$ is severely biased toward zero.

BZ's approach of continuous-time model estimation based on moments of daily realized measures has been followed in several studies, and the present paper belongs to this strand of the literature. Corradi and Distaso (2006), hereafter referred to as CD, proposed to use unconditional moments and autocovariances of the realized variance

\footnotetext{
${ }^{4}$ See, for example, Andersen and Bollerslev (1997), who documented empirical evidence of pervasive intraday periodicity in asset return volatility.
} 
and related realized measures for a similar GMM estimation procedure, but they did not consider the estimation of $\rho . \quad \mathrm{BZ}$ and $\mathrm{CD}$ used only the high frequency observations of the price process. Garcia et al. (2011), hereafter referred to as GLPR, proposed to use two sets of moment conditions, including higher-order ones, for GMM estimation of the Heston model: one set involving the daily asset returns and daily realized measures calculated using high-frequency intraday asset returns, and another set involving the daily asset returns and the daily observations of the model-dependent implied volatility. As with this paper, one focus was on the estimation of $\rho$. The finite sample simulation results provided in GLPR and in this paper indicate that the GLPR estimator provides a vast improvement over the BZ estimator in terms of accuracy in estimating $\rho$ of the Heston SV model with nonzero leverage. We demonstrate by simulation that the use of high frequency intraday implied volatility index data would lead to further substantial gains in efficiency.

The CBOE's S\&P 500 implied (or expected) volatility index (VIX) is designed to measure the volatility of the S\&P 500 index without relying on a particular option pricing model, such as the Black-Scholes or Heston models. Many authors have attempted to exploit information in VIX in estimating models for the S\&P 500 index. Under the assumption that the S\&P 500 index follows an affine-drift SV process (possibly with certain types of jumps), VIX is an affine transformation of its spot variance. We are not the first to take advantage of this relation. Based on this relation, Duan and Yeh (2010) proposed an estimator for the affine-drift CEV SV model with Poisson-type price jumps for the S\&P 500 index, using daily observations of both the S\&P 500 index and VIX. However, they only used daily data to estimate a discretized version of the model. Ait-Sahalia and Kimmel (2007) also used the VIX jointly with the S\&P 500 data in implementing their approximate maximum likelihood estimator. Bakshi et al. (2006) and Dotsis et al. (2007), among others, take the VIX process as the object of direct interest rather than treating it as an instrument to estimate the underlying volatility process, and used daily VIX observations to estimate the continuous-time SV models for VIX. However, none of these studies used data of frequencies higher than a day. 
Applicability of the proposed approach is not limited to the S\&P 500 index. If there exists a liquid options market for the underlying process of our interest, with a wide spectrum of strike prices, and the intraday high frequency data of their prices were available, we may calculate the "model-free" implied volatility values at a high enough frequency for the application of our proposed approach. For many financial series, the implied volatility calculation step is conveniently done by exchanges and other institutions. On the heels of the success of VIX, the universe of "model-free" implied volatility indices, as well as exchange-traded options and futures on these volatility indices, has been expanding rapidly in recent years.

The CBOE now calculates and disseminates volatility-related indices for a variety of financial market indices, and currency and commodities ETFs, including the CBOE NASDAQ-100 Volatility Index, CBOE EuroCurrency Volatility Index, CBOE Crude Oil Volatility Index, CBOE Gold ETF Volatility Index. The CBOE and the Chicago Mercantile Exchange (CME) Group work together to provide the CBOE/NYMEX Crude Oil (WTI) Volatility Index and CBOE/COMEX Gold Volatility Index, applying the CBOE VIX methodology to the prices of options on crude oil and gold futures. They also intend to provide the CBOE/CBOT Soybean Volatility Index and Corn Volatility Index. The Deutsche Börse provides the VDAX-NEW index for the DAX, and Osaka University, Japan, provides the VXJ and CSFI-VXJ for the Nikkei 225 index (see Fukasawa et al. (2010) for the latter indices). Various institutions calculate and update "model-free" implied volatility indices for other indices, although the updating frequency is not always high enough for our purpose.

Another contribution of this paper is a proper adjustment of the moment conditions to reflect the fact that daily realized measures are calculated only for the trading hours that do not cover a full day. In estimating the Heston model for share prices of individual stocks or the S\&P 500 index, CD and GLPR treat the six and a half hours (9:30 am 4:00 pm) for which NYSE is open as a full day as if overnight hours were non-existent. Their closed-form moment conditions for the Heston SV case clearly need to be modified, considering the overnight market closure (nearly three quarters of a day). 
Otherwise, the estimator will be biased. We corroborate this claim by first driving the modified moment conditions allowing for overnight market closure, and then performing Monte Carlo simulation using the BZ moment conditions.

The plan of the remainder of the paper is as follows. Section 2 develops a framework for using intraday high frequency implied volatility indexes data for SV model estimation, in particular, a leverage estimator using realized measures of price and volatility indexes for the Heston SV special case. Section 3 presents some finite sample simulation results. Section 4 analyzes the empirical results using intraday high frequency S\&P 500 and VIX. Section 5 gives some concluding remarks.

\section{Estimation of leverage and other parameters using realized measures of both the price and implied volatility index}

Consider the following class of affine-drift SV diffusion processes:

$$
\begin{gathered}
d p_{t}=\sqrt{V_{t}} d B_{t}, \\
d B_{t}=\rho d W_{t}^{(1)}+\sqrt{1-\rho^{2}} d W_{t}^{(2)}, \\
d V_{t}=\kappa\left(V_{t}-\theta\right) d t+\sigma\left(V_{t}\right) d W_{t}^{(1)},
\end{gathered}
$$

where $p_{t}$ is the log price process, $W_{t}^{(1)}, W_{t}^{(2)}$ are Brownian motions independent of each other, and $V_{t}$ is called the spot variance process ${ }^{5}$. The parameters $\kappa$ and $\theta$ determine, respectively, the speed of variance mean reversion and the average level of the spot variance. As $d B_{t} d W_{t}^{(1)}=\rho d t, \quad \rho$ is the so-called leverage parameter. When the diffusion coefficient $\sigma\left(V_{t}\right)$ of the variance process (3) is of the form $\sigma V_{t}^{\gamma}$ with $\sigma>0$, it is called the affine-drift CEV diffusion. The affine-drift CEV with $\gamma=0.5$ is Heston's (1993) square-root SV model, and the affine-drift CEV with

\footnotetext{
${ }^{5}$ The drift function of the price equation (1), which is irrelevant for our analysis, is set to be zero, as in BZ.
} 
$\gamma=1.0$ is Nelson's (1990) GARCH SV diffusion.

A key element in constructing the proposed estimator is the well-known fact that, for the above SV model, the following relation holds between the risk-neutral expectation of the integrated variance over any horizon, $\tau>0$ :

$$
v_{t, t+\tau}:=E_{t}^{Q}\left[\int_{t}^{t+\tau} V_{s} d s\right]=\lambda V_{t}+\delta
$$

at each point in time, where $\lambda>0$ and $\delta$ are constants that depend on $\tau$ and the parameters of the model, both under the physical and risk-neutral measures (see, for example, Duan and Yeh (2010)). $E_{t}^{Q}[\square]$ is the expectation operator under the risk neutral measure, conditional on the filtration on $\mathrm{F}_{t}$, and $\left\{\mathrm{F}_{t}\right\}$ is the filtration on the probability space on which $\left\{p_{t}\right\}$ is defined ${ }^{6}$. Note that it is the affine form of the drift function, rather than a particular form of the diffusion function (such as the square-root diffusion function in the Heston SV model), of the spot variance process that gives rise to the affine relation, namely equation (4).

The VIX index, a widely watched stock market volatility indicator that was introduced by the Chicago Board Options Exchange (CBOE), is intended to approximate $\sqrt{v_{t, t+\tau}}$ at $\tau=30$, of the S\&P 500 index process, using the theoretical formula in the model-free implied volatility literature (see Britten-Jones and Neuberger (2000), Demeterfi et al. (1999), Jian and Tian (2005)), linking the market prices of a cross-section of options on the S\&P 500 index and $v_{t, t+\tau}$ (see CBOE (2009)). In the discussion below, we fix $\tau=30$, write $v_{t}$ for $v_{t, t+30}$, and treat $V I X_{t}^{2}=v_{t}$ as an exact relationship ${ }^{7}$, which makes the spot variance observable up to an affine transformation

\footnotetext{
${ }^{6}$ An affine relation, albeit with different values of the two constants, between the expectation of the integrated variance and the spot variance, holds under the physical measure as well, which is widely used in deriving analytical expressions for some of the moment conditions by BZ, GLPR, and this paper.

Aitt-Sahalia and Kimmel (2007) simply used VIX to approximate the left-hand side of equation (4)
} 
with unknown parameters $\lambda$ and $\delta$.

For the S\&P 500 index, the CBOE calculates and disseminates the VIX index on a real-time and intraday very high frequency basis, so that we do not have to collect $\mathrm{S} \& \mathrm{P}$ 500 index options tick data for the calculation of $v_{t}$. If there is a liquid market for options written on the process of interest, with a reasonably wide and dense cross-section of strikes, a VIX-type model-free implied volatility may be calculated for financial instruments other than the S\&P 500 index. If high frequency observations of the price process and a VIX-type index, or option prices necessary to calculate such an index, were available, the realized leverage could be calculated. Hence, the discussion below also applies to financial processes in addition to the S\&P 500 index. In the empirical section, we use intraday VIX data.

Define

$$
\mathrm{V}_{t, T}(q):=\int_{t}^{T} V_{s}^{q} d s
$$

and

$$
\mathrm{V}_{t, T}:=\mathrm{V}_{t, T}(1)
$$

Under the SV model, equations (1)-(3), the realized variance $R V_{t, T}$ for the time interval $[t, T]$ is such that:

$$
R V_{t, T}:=\sum_{i=1}^{N}\left(p_{t+i(T-t) / N}-p_{t+(i-1)(T-t) / N}\right)^{2} \stackrel{p}{\rightarrow} \mathrm{V}_{t, T}
$$

where $\stackrel{p}{\rightarrow}$ denotes convergence in probability as the number of observations, $N$, during the fixed time interval $[t, T]$ goes to infinity.

without invoking the theory of the model-free implied variance. 
For estimating the parameters of the SV model, BZ and CD suggested using moments of the daily realized variance ${ }^{8}$ (BZ also suggested adding the cross moment of the daily $\log$ index level, $p_{t}$, and the daily realized variance for estimating $\rho$ jointly with the other parameters of the Heston model), and GLPR recommended using higher-order moments of the daily realized variance or daily (model-dependent) implied volatility. For estimating $\rho$ jointly, GLPR proposed adding the (higher-order) cross moments of the daily $\log$ return $p_{t}-p_{t-1}$ (rather than the log level $p_{t}$ ) and the realized variance (or the implied volatility), which is notably different from BZ's choice of the cross moment.

Essentially, our new proposal uses various moments of the realized measures of the model-free implied volatility index and the realized covariation/correlation between the underlying index and the model-free implied volatility index, defined below, for conducting statistical inference on the SV process ${ }^{9}$. In order to substantiate our claim about the benefit of using high frequency implied volatility data, we will focus on the Heston SV example. For SV models other than the Heston SV model, we can use the simulated methods of moment (SMM) estimator with moments of the realized measures calculated by simulation since analytical expressions for the moments are, in general, unavailable ${ }^{10}$.

We have for the realized variance, $R V V_{t, T}$, of $v_{t}$, and the realized covariation, $\operatorname{RCOV}_{t, T}$, between $p_{t}$ and $v_{t}$ :

$$
R V V_{t, T}:=\sum_{i=1}^{N}\left(v_{t+i(T-t) / N}-v_{t+(i-1)(T-t) / N}\right)^{2} \stackrel{p}{\rightarrow} \lambda^{2} \int_{t}^{T} \sigma^{2}\left(V_{s}\right) d s
$$

\footnotetext{
${ }^{8} \mathrm{CD}$ also suggested versions with other realized measures, such as the realized bipower variation replacing the realized variance.

9 One could also use realized power variations other than the realized variance.

${ }^{10}$ It may not be computationally feasible to calculate the conditional moments at each point in time for each iteration of the objective function minimization. The SMM estimator of CD based on unconditional moments (including autocovariances) may be used instead.
} 


$$
\operatorname{RCOV}_{t, T}:=\sum_{i=1}^{N}\left(v_{t+i(T-t) / N}-v_{t+(i-1)(T-t) / N}\right)\left(p_{t+i(T-t) / N}-p_{t+(i-1)(T-t) / N}\right) \stackrel{p}{\rightarrow} \lambda \rho \int_{t}^{T} \sigma\left(V_{s}\right) V_{s}^{1 / 2} d s
$$

We can also define the realized correlation, or the realized leverage, as follows:

$$
\operatorname{RCORR}_{t, T}:=\frac{\operatorname{RCOV}_{t, T}}{\sqrt{R V V_{t, T}} \sqrt{R V_{t, T}}} \stackrel{p}{\rightarrow} \rho_{t}^{*}:=\rho \frac{\int_{t}^{T} \sigma\left(V_{s}\right) V_{s}^{1 / 2} d s}{\sqrt{\mathrm{V}_{t, T} \int_{t}^{T} \sigma^{2}\left(V_{s}\right) d s}}
$$

Note that $\lambda$ is cancelled out. If we assume that the volatility of the spot variance is of the CEV function, $\sigma\left(V_{t}\right)=\sigma V_{t}^{\gamma}$, we obtain $R V V_{t, T} \stackrel{p}{\rightarrow} \lambda^{2} \sigma^{2} V_{t, T}(2 \gamma)$, $\operatorname{RCOV}_{t, T} \stackrel{p}{\rightarrow} \lambda \rho \sigma \mathrm{V}_{t, T}\left(\gamma+\frac{1}{2}\right)$, and $\rho_{t}^{*}:=\rho \mathrm{V}_{t, T}\left(\gamma+\frac{1}{2}\right) / \sqrt{\mathrm{V}_{t, T}(2 \gamma) \mathrm{V}_{t, T}}$. Note that $\sigma$ is cancelled out further. For the special case of the Heston SV process (the affine drift CEV SV with $\gamma=0.5), \quad \rho_{t}^{*}=\rho$, thereby leading to a key result ${ }^{11}$ :

$$
\operatorname{RCORR}_{t, T} \stackrel{p}{\rightarrow} \rho .
$$

This means that, in the continuous-record limit with a fixed $T$, the leverage parameter can be recovered without statistical uncertainty if the Heston SV specification is correct.

The consistency result (11) may not hold in reality due to a variety of factors, such as microstructure noise, and the relation, $\operatorname{RCORR}_{t, T}=\rho$, is not exact, hence is not a deterministic constraint, for finite $N$ and $T$, even in the absence of microstructure noise. Nevertheless, $R C O R R_{t, T}$ may perform well as an estimator of the leverage parameter, $\rho$, under the Heston SV process. The interval over which the quantities are measured at high frequency is defined to be $[t, T]$ in the above, for notational simplicity. However, (11) clearly holds when the measurement period is a collection of

${ }^{11}$ For the Heston SV case, the central limit theorem also obtains (as $N \rightarrow \infty$ with $T$ fixed) as a straightforward application of Theorem 2 of Barndorff-Nielsen et al. (2006):

$$
\frac{\sqrt{N}\left(R_{\left.C O R R_{t, T}-\rho\right)}\right.}{\sqrt{\mathrm{V}_{t, T}(2)} / \mathrm{V}_{t, T}} \stackrel{\text { Law }}{\rightarrow} \mathrm{N}\left(0,\left(1+\rho^{2}\right)\right)
$$


subintervals $\left[t_{1}, t_{2}\right], \quad\left[t_{3}, t_{4}\right], \cdots, \quad\left[t_{K-1}, t_{K}\right]$, where $t=t_{1}<t_{2} \quad<t_{3} \quad<t_{4} \quad<\cdots$ $<t_{K-1}<t_{K}=T$ if the three realized measures that comprise the realized correlation are defined over the same set of subintervals and the observation intervals shrink to zero in each subinterval. This is convenient as most financial markets have interruptions in trading, such as overnight hours, holidays, and weekends.

For non-Heston cases, we recommend using moments of the realized covariance or correlation, and estimate $\rho$, jointly with the other parameters by GMM or SMM, as the stochastic quantity $\rho_{t}^{*}$, to which $R C O R R_{t, T}$ converges in probability, is not equal to $\rho$, and $\left|\rho_{t}^{*}\right|<|\rho|$ holds as $\int_{t}^{T} \sigma\left(V_{s}\right) V_{s}^{1 / 2} d s<\sqrt{\mathrm{V}_{t, T} \int_{t}^{T} \sigma^{2}\left(V_{s}\right) d s}$, by the Cauchy-Schwartz inequality. However, we report simulation results in the next section where, for the affine-drift CEV case, $\operatorname{RCORR}_{t, T} \approx \rho$, even when $\gamma=1$ or $\gamma=1.5$.

We also have:

$$
R V V_{t, T} / R V_{t, T} \stackrel{p}{\rightarrow} \lambda^{2} \int_{t}^{T} \sigma^{2}\left(V_{s}\right) d s / \mathrm{V}_{t, T}
$$

for fixed $[t, T]$, the right-hand side of which becomes $\lambda^{2} \sigma^{2} \mathrm{~V}_{t, T}(2 \gamma) / \mathrm{V}_{t, T}$ under the affine-drift CEV SV and $\lambda^{2} \sigma^{2}$ (a constant) under the Heston SV model. For estimating the Heston SV model, this high frequency (with a fixed-T) asymptotic relation should be particularly helpful if $\lambda$ is to be estimated jointly. This is so because, as is the case with estimating $\rho$ by the realized leverage, $\lambda \sigma$ for the Heston SV model can be recovered without statistical imprecision in the continuous-record limit for fixed $T$, under ideal conditions.

Furthermore, we have the following results involving $\lambda$ and $\delta$ :

$$
\bar{v}_{t, T}(1) \stackrel{p}{\rightarrow} \lambda \mathrm{V}_{t, T}+(T-t) \delta
$$




$$
\bar{v}_{t, T}(2) \stackrel{p}{\rightarrow} \lambda^{2} \mathrm{~V}_{t, T}(2)+2 \lambda \delta \mathrm{V}_{t, T}+(T-t) \delta^{2}
$$

where

$$
\bar{v}_{t, T}(q):=\frac{T-t}{N} \sum_{i=1}^{N} v_{t+i(T-t) / N}^{q} .
$$

These results may be exploited in joint estimation for $(\kappa, \theta, \sigma, \rho, \lambda, \delta)$. The additional parameters, $\lambda$ and $\delta$, may be informative about the parameters of the SV process under the risk-neutral measure, and hence also the volatility risk premium, but are nuisance parameters if the interest is only in estimating the parameters $(\kappa, \theta, \sigma, \rho)$ of the SV model under the physical measure. In this paper, we do not pursue the use of these relations further.

In the remainder of the paper, we focus on the Heston SV example, and consider extending the BZ estimator ${ }^{12}$. BZ showed that, for the special case of the Heston SV model where the variance diffusion is given by ${ }^{13}$ :

$$
d V_{t}=\kappa\left(V_{t}-\theta\right) d t+\sigma \sqrt{V_{t}} d W_{t}^{(1)}
$$

the following analytical expressions for the conditional moments of $V_{t, t+1}$ hold:

$$
\begin{gathered}
E_{t}\left[\mathrm{~V}_{t+1, t+2}\right]=\alpha_{1} E_{t}\left[\mathrm{~V}_{t, t+1}\right]+\beta_{1} \\
E_{t}\left[\mathrm{~V}_{t+1, t+2}^{2}\right]=\alpha_{1}^{2} E_{t}\left[\mathrm{~V}_{t, t+1}^{2}\right]+I_{1} E_{t}\left[\mathrm{~V}_{t, t+1}\right]+J_{1} \\
E_{t}\left[p_{t+1} \frac{\mathrm{V}_{t+1, t+2}-b_{1}}{a_{1}}\right]=\alpha_{1} E_{t}\left[p_{t} \frac{\mathrm{V}_{t, t+1}-b_{1}}{a_{1}}\right]+\left(1-\alpha_{1}\right)\left(p_{t}+\frac{\rho \sigma}{\kappa}\right) \theta
\end{gathered}
$$

\footnotetext{
${ }^{12}$ Alternatively, one can take the GLPR estimator with higher-order moments as a starting point, but making non-full-day adjustments to the analytical expressions for the higher-order conditional moments would be more involved.

${ }^{13}$ Note that these equations reflect the corrections by Bollerslev and Zhou (2004) for the original equations in BZ.
} 


$$
+a_{1} \rho \sigma\left(E_{t}\left[p_{t} \frac{\mathrm{V}_{t, t+1}-b_{1}}{a_{1}}\right]-\theta\right)
$$

where

$$
\begin{gathered}
\alpha_{\Delta}:=e^{-\kappa \Delta}, \beta_{\Delta}:=\theta\left(1-\alpha_{\Delta}\right), \\
a_{\Delta}:=\kappa^{-1}\left(1-e^{-\kappa \Delta}\right), b_{\Delta}:=\theta\left(\Delta-a_{\Delta}\right), \\
A_{\Delta}:=\frac{\sigma^{2}}{\kappa^{2}}\left[\kappa^{-1}\left(1-e^{-2 \kappa \Delta}\right)-2 e^{-\kappa \Delta} \Delta\right], \\
B_{\Delta}:=\frac{\sigma^{2} \theta}{\kappa^{2}}\left[\left(1+2 e^{-\kappa \Delta}\right) \Delta-\frac{1}{2 \kappa}\left(e^{-\kappa \Delta}+5\right)\left(1-e^{-\kappa \Delta}\right)\right], \\
C_{\Delta}:=\frac{\sigma^{2}}{\kappa}\left(e^{-\kappa \Delta}-e^{-2 \kappa \Delta}\right), D_{\Delta}:=\frac{\sigma^{2} \theta}{2 \kappa}\left(1-e^{-\kappa \Delta}\right)^{2}, \\
I_{1}:=a_{1}\left(C_{1}+2 \alpha_{1} \beta_{1}\right)+\alpha_{1}\left(1-\alpha_{1}\right)\left(2 b_{1}+a_{1}^{-1} A_{1}\right), \\
J_{1}:=-I_{1} b_{1}+a_{1}^{2}\left(D_{1}+\beta_{1}^{2}\right)+\left(2 a_{1} b_{1}+A_{1}\right) \beta_{1}+\left(1-\alpha_{1}^{2}\right)\left(b_{1}^{2}+B_{1}\right) .
\end{gathered}
$$

and $E_{t}[\square]:=E\left[\square \mid \mathrm{G}_{l}^{\mathrm{l}}\right]$ is the expectation operator under the physical measure, conditional on $\mathrm{G}_{l}^{\Delta}:=\sigma\left\{\mathrm{V}_{s-1, s-1+\Delta} ; s=t, t-1, \cdots\right\}$, the discrete filtration, or the sigma algebra generated by the daily realized variance series. The notation $\mathrm{G}^{\Delta}$ is introduced here in preparation for the case of non-full-day trading sessions. For estimating $(\kappa, \theta, \sigma, \rho)$ of the Heston $\mathrm{SV}, \mathrm{BZ}$ proposed a GMM estimator (GMM-BZ1), using the sample analogues of the following set of moment conditions:

$$
\begin{gathered}
E\left[E_{t}\left[\mathrm{~V}_{t+1, t+2}\right]-\mathrm{V}_{t+1, t+2}\right]=0 \\
E\left[E_{t}\left[\mathrm{~V}_{t+1, t+2}^{2}\right]-\mathrm{V}_{t+1, t+2}^{2}\right]=0 \\
E\left[\left(E_{t}\left[\mathrm{~V}_{t+1, t+2}\right]-\mathrm{V}_{t+1, t+2}\right) \mathrm{V}_{t-1, t}\right]=0 \\
E\left[\left(E_{t}\left[\mathrm{~V}_{t+1, t+2}^{2}\right]-\mathrm{V}_{t+1, t+2}^{2}\right) V_{t-1, t}\right]=0 \\
E\left[\left(E_{t}\left[\mathrm{~V}_{t+1, t+2}\right]-\mathrm{V}_{t+1, t+2}\right) \mathrm{V}_{t-1, t}^{2}\right]=0 \\
E\left[\left(E_{t}\left[\mathrm{~V}_{t+1, t+2}^{2}\right]-\mathrm{V}_{t+1, t+2}^{2}\right) \mathrm{V}_{t-1, t}^{2}\right]=0
\end{gathered}
$$




$$
E\left[E_{t}\left[p_{t+1} \frac{\mathrm{V}_{t+1, t+2}-b_{1}}{a_{1}}\right]-p_{t+1} \frac{\mathrm{V}_{t+1, t+2}-b_{1}}{a_{1}}\right]=0
$$

where the unconditional expectation $E[\square]$ is taken under the physical measure. BZ suggested simulations for calculating the conditional moments if the model being estimated is a non-Heston SV and suitable closed-form expressions are not available. As reported in the next section, the results of our Monte Carlo simulation experiments indicate that $\rho$, when estimated jointly by $G M M-B Z 1$ with the other parameters of the Heston SV, is severely biased.

For estimating $(\kappa, \theta, \sigma)$ of the Heston SV model, we propose two new methods, namely: $(i)$ estimate all the parameters $(\kappa, \theta, \sigma)$ and $\rho$ jointly by GMM using the sample analogues of equations (22) - (27) and the realized leverage formula:

$$
\operatorname{RCORR}_{t, T}-\rho
$$

replacing the sample analogue of (28) (called $G M M-B Z-R L$ ); or (ii) estimate $\rho$ by $\hat{\rho}=R C O R R_{t, T}$ (called $\left.\hat{\rho} R L\right)$ and $(\kappa, \theta, \sigma)$ by BZ's original GMM estimator, using the sample analogues of equations (22) - (27) (called GMM-BZ2).

It may be possible to derive the conditions for the SV process, the measurement error process, and the relative rate of $N \rightarrow \infty$ and $T \rightarrow \infty$, under which the estimators, $G M M-B Z-R L$ and $R L$, are consistent, along the lines of CD. The realized bipower variation counterparts $R B V_{t, T}$ and $R B V V_{t, T}$ to $R V_{t, T}$ and $R V V_{t, T}$, respectively, are jump-robust estimators for $\bigvee_{t, T}$ and $\int_{t}^{T} \sigma^{2}\left(V_{s}\right) d s$ even if the price (1) and the spot variance process (3) contain certain types of jumps. CD proposed to use $R B V_{t, T}$ for a jump-robust specification test of the diffusion components of a jump-diffusion model. $R C O V_{t, T}$ may be affected, even asymptotically $(N \rightarrow \infty)$, by jumps if the price jumps and volatility jumps arrive simultaneously (see Jacod and Todorov (2010) for empirical 
evidence of price-volatility cojumps in the S\&P 500 index). It is also possible to construct a realized measure that serves as a jump-robust estimator for $\int_{t}^{T} \rho V_{s}^{1 / 2} \sigma\left(V_{s}\right) d s$. We may also use a Lee-Mykland-type estimator (Lee and Mykland (2008)) to estimate directly and remove jumps from the observations. We leave these as topics for future research ${ }^{14}$.

A major complication in estimating a model of a financial process is that high frequency intraday observations used for constructing realized measures often do not cover an entire trading day. For example, the S\&P 500 cash index value is observed only for the period 9:30-16:00 per trading day, which is less than one-third of a day. In applying GMM estimators with sets of moment conditions involving realized measures to individual stock prices and the S\&P 500 index, CD and GLPR ignore the existence of overnight non-trading hours. Treating 6.5-hour daily realized measures as if they were 24-hour flow quantities, and ignoring the evolution of the price and its stochastic volatility processes during overnight hours, lead to incorrect analytical expressions for the moments as functions of the unknown parameters and observables.

Hence, we modify equations (22) - (27) as follows, taking the market closure (16:00-9:30) into consideration:

$$
\begin{gathered}
E\left[E_{t}^{\Delta}\left[\mathrm{V}_{t+1, t+1+\Delta}\right]-\mathrm{V}_{t+1, t+1+\Delta}\right]=0 \\
E\left[E_{t}^{\Delta}\left[\mathrm{V}_{t+1, t+1+\Delta}^{2}\right]-\mathrm{V}_{t+1, t+1+\Delta}^{2}\right]=0 \\
E\left[\left(E_{t}^{\Delta}\left[\mathrm{V}_{t+1, t+1+\Delta}\right]-\mathrm{V}_{t+1, t+1+\Delta}\right) \mathrm{V}_{t-1, t-1+\Delta}\right]=0 \\
E\left[\left(E_{t}^{\Delta}\left[\mathrm{V}_{t+1, t+1+\Delta}^{2}\right]-\mathrm{V}_{t+1, t+1+\Delta}^{2}\right) \mathrm{V}_{t-1, t-1+\Delta}\right]=0 \\
E\left[\left(E_{t}^{\Delta}\left[\mathrm{V}_{t+1, t+1+\Delta}\right]-\mathrm{V}_{t+1, t+1+\Delta}\right) \mathrm{V}_{t-1, t-1+\Delta}\right]=0 \\
E\left[\left(E_{t}^{\Delta}\left[\mathrm{V}_{t+1, t+1+\Delta}^{2}\right]-\mathrm{V}_{t+1, t+1+\Delta}^{2}\right) \mathrm{V}_{t-1, t-1+\Delta}\right]=0 .
\end{gathered}
$$

\footnotetext{
${ }^{14}$ We would need to carefully consider how the spot/implied volatility relation, equation (4), is affected by various types of jumps.
} 
where $E_{t}^{\Delta}[\square]:=E\left[\square \mid \mathrm{G}_{i}^{\Delta}\right]$. For example, observations of the S\&P 500 index for a trading day are from time period $t(9: 30)$ to $t+\Delta \quad(16: 00)(\Delta \approx 0.27)$. Conditioning on the information available at the session's opening, rather than the session's closing, makes the derivation of conditional moments and the resulting expressions much simpler.

Following the derivation of equations (17) and (18) by BZ for the case of $\Delta=1$ (24-hour trading), it is straightforward to obtain:

$$
\begin{gathered}
E_{t}^{\Delta}\left[\mathrm{V}_{t+1, t+1+\Delta}\right]=\alpha_{1} E_{t}^{\Delta}\left[\mathrm{V}_{t, t+\Delta}\right]+\beta_{1} \Delta \\
E_{t}^{\Delta}\left[\mathrm{V}_{t+1, t+1+\Delta}^{2}\right]=\alpha_{1}^{2} E_{t}^{\Delta}\left[\mathrm{V}_{t, t+\Delta}^{2}\right]+I_{\Delta} E_{t}^{\Delta}\left[\mathrm{V}_{t, t+\Delta}\right]+J_{\Delta}
\end{gathered}
$$

where

$$
\begin{aligned}
& I_{\Delta}:=a_{\Delta}\left(C_{1}+2 \alpha_{1} \beta_{1}\right)+\alpha_{1}\left(1-\alpha_{1}\right)\left(2 b_{\Delta}+a_{\Delta}^{-1} A_{\Delta}\right), \\
& J_{\Delta}:=-I_{\Delta} b_{\Delta}+a_{\Delta}^{2}\left(D_{1}+\beta_{1}^{2}\right)+\left(2 a_{\Delta} b_{\Delta}+A_{\Delta}\right) \beta_{1}+\left(1-\alpha_{1}^{2}\right)\left(b_{\Delta}^{2}+B_{\Delta}\right) .
\end{aligned}
$$

See the appendix for a derivation of the above relationships.

Note that, although equations (36) and (37) appear to be virtually identical to BZ's 24-hour trading versions, namely equations (17) and (18), they are different. The $I_{\Delta}$ and $J_{\Delta}$ given above are modifications of BZ's $I$ and $J$, allowing for non-full-day trading sessions over which the integrated variance is defined, and the resulting time gap between $t+\Delta$ (the end of the period over which $\mathrm{V}_{t, t+\Delta}$ is defined) and $t+1$ (the beginning of the period over which $\mathrm{V}_{t+1, t+1+\Delta}$ is defined). If $\Delta=1$, equations (36) and (37) reduce to (17) and (18), respectively.

We may use the sample analogues of equations (30) - (35) and $\sum_{t=1}^{T} \frac{R C O V_{t, t+\Delta}}{\sqrt{R V V_{t, t+\Delta}} \sqrt{R V_{t, t+\Delta}}}-\rho$ in constructing moment conditions for GMM estimation. Our adjustment method 
assumes that the spot variance follows the same Heston SV during trading hours and overnight hours. Admittedly, this is an unrealistic assumption. We could consider lowering the average variance for night hours, but leave it as a future research topic. Delving too deeply into seasonality issues would defeat the purpose of using daily aggregate quantities for estimating simple continuous-time SV models that have proved useful as approximations of financial processes at the daily or weekly measurement intervals. Finally, note that the realized leverage without any adjustment for $\Delta<1$ converges to $\rho$ in probability, even if the spot variance process follows the Heston SV with different sets of $(\kappa, \theta, \sigma)$ values during trading hours and during overnight non-trading hours, if $\rho$ remains the same.

\section{Finite sample simulation results}

In this section, we report the results of Monte Carlo simulation experiments to examine the finite sample properties of the BZ GMM estimator and the proposed estimators for the Heston model for the case $\rho \neq 0$. Note that, although BZ derived moment conditions for the Heston SV model with $\rho \neq 0$, and extended the results to the Heston SV model with price jumps and two-factor SV models, they only conducted their experiments for the case of the Heston SV model with zero leverage, $\rho=0$.

The sample paths of $p_{t}$ and $V_{t}$ are simulated by the Euler-Maruyama scheme ( $\Delta t=1 / 2880$, or 30 seconds) 10,000 times. The length, $T$, in days of each simulated path is 960, as in GLPR, after the observations from a burn-in period of 240 days are discarded. At the start of the burn-in period, $V_{t}$ is set to $\theta$, the long-run average of the spot variance. As in BZ and GLPR, the unit time is a day rather than a year. Note that we do not observe $V_{t}$, in practice. However, as we treat its affine transformation, $v_{t}$, as observable under the affine-drift SV process and the extra parameters, $\lambda$ and $\delta$, of the transformation are cancelled out in the realized leverage that is calculated using observations of $p_{t}$ and $v_{t}$, the simulation results would be the 
same if we were to use observations of $v_{t}=\lambda V_{t}+\delta$, regardless of the values assigned for $\lambda$ and $\delta$. Hence, in order to simplify the experiments, we choose $\lambda=1$ and $\delta=0$, thereby making $V_{t}$ observable.

\section{(i) Data from contiguous full-day trading sessions}

We first examine the scheme in which daily trading sessions last 24 hours and there are no breaks between daily sessions. The values of $p_{t}$ and $v_{t}=V_{t}$ are observed once every five minutes, and daily realized measures are calculated once a day, using 288 five-minute $\log$ price returns and differences in $V_{t}$. GLPR used a simulation scheme that is similar to ours, but they divided each day into 80 "5-minute" observation intervals, which are effectively 18-minute intervals.

The first set of true parameter values is $(\kappa, \theta, \sigma, \rho)=(.1, .25, .1,-.5)$, which corresponds to Parameter Set A in GLPR. The long run spot variance, $\theta=.25$, which is the value set in BZ, is about $7.75 \%$ per annum if one year has 240 days. The second set of true parameter values is identical to the first, except that $\kappa=.05$, which induces a slower mean reversion of the spot variance. The parameters are estimated using observations from each of the simulated sample paths of $\left\{p_{t}\right\}$ or $\left\{p_{t}, V_{t}\right\}$. For all of our GMM estimators, we use the optimal covariance matrix estimated by the Newey-West scheme with five lags, as in BZ, and impose the stationarity condition, $2 \kappa \theta>\sigma^{2}$.

The results for the first set of true parameters are summarized in Panel A of Table 1. Note that the biases and RMSEs shown are multiplied by 100 . Both the bias and the

RMSE of $\hat{\rho}$ and, to a lesser but still serious degree, the RMSE of $\hat{\theta}$ of GMM-BZ1, are so large as to render $G M M-B Z 1$ meaningless and undesirable. The performance of the proposed estimator $G M M-B Z 1-R L$ relative to $G M M-B Z 1$ is better overall in terms of biases and RMSEs, except for a slightly larger bias in estimating $\kappa$, and is vastly 
superior as an estimator of $\rho$. In fact, the bias and RMSE of $\hat{\rho}$ are only .0007 and .0016, respectively, which are negligible compared with those produced by $G M M-B Z 1$. More importantly, the performance of $G M M-B Z 1-R L$ in estimating $\rho$ appears to be substantially better than the GLPR estimators ${ }^{15}$.

As $\rho$ does not enter equations (22) - (27), and the other parameters do not enter (29), there may be no efficiency gains in estimating $\rho$ jointly with the other parameters by GMM rather than separately by using the realized leverage. This, in fact, appears to be the case, as is corroborated by $R L$ 's even smaller bias and RMSE as an estimator of $\rho$. The performance of $G M M-B Z 2$ as an estimator of $(\kappa, \theta, \sigma)$ is comparable to that of $G M M-B Z-R L$. The overall pattern in the results of the experiment using the second set of true parameter values is similar to the previous case.

\section{(ii) Data from non-full-day trading sessions}

In this subsection, we investigate the effects on the GMM estimator of not properly correcting the moment conditions for the existence of market closure between trading sessions. We assume that the log price and the variance follow the Heston SV process, with $(\kappa, \theta, \sigma, \rho)=(.1, .25, .1,-.5)$, both day and night, and are observed only for the first six hours of each day. We assume that the econometrician treats the observed six hours of data as arising from the first $h$ hours of each day $(h=6,12,18$, or 24$)$, sets $\Delta=h / 24$, and estimates $(\kappa, \theta, \sigma)$ by GMM, with the sample analogues of the moment conditions in equations (30) - (35), and $\rho$ by the realized leverage. It is noted that $h=6$ is correct, and $h=24$ ignores 18 hours in between sessions, in addition to incorrectly treating 6 hours as 24 hours. Note that no adjustment is required in computing the realized leverage for the cases with $\Delta<1$.

${ }^{15}$ Note, however, that a day is divided into 288 subintervals in our scheme, while there are only 80 in theirs. On a separate issue, a comparison of our simulation results for GMM-BZ1 and GLPR's results for their estimators reveals that the efficiency gains of the GLPR estimators, relative to $G M M-B Z 1$, likely arise not only because of the use of implied volatilities, but also through the use of higher-order moments and/or the use of asset returns (rather than asset prices) in constructing cross moments. 
The results are shown in Table 2. The biases and RMSEs increase as $h$ deviates from $h=6$, except for $\kappa$. Our simulation results indicate that there is a serious need for adjustments.

(iii) Realized leverage under the affine-drift CEV with $\gamma \neq 0.5$

In this subsection, we report the results of Monte Carlo simulation experiments for the realized leverage as an estimator of $\rho$, when the affine-drift CEV SV, equations (1) (3) with $\sigma V_{t}^{\gamma}, \gamma \neq .5$, generates the data $\left\{p_{t}, v_{t}\right\}^{16}$. This exercise is to check the robustness of the realized leverage estimator for $\rho$ under the misspecification of Heston SV model's square-root diffusion function. The results should not be construed as our recommendation for the realized leverage as a direct $\rho$ estimator for non-Heston cases. Recall that the realized leverage under the affine-drift CEV converges in probability to $\rho \mathrm{V}_{t, T}\left(\gamma+\frac{1}{2}\right) / \sqrt{\mathrm{V}_{t, T}(2 \gamma) \mathrm{V}_{t, T}}$, the absolute value of which is smaller than the true $|\rho|$, unless $\gamma=.5$. The setup is identical to the first setup ( $\kappa=.1$ ) used for the contiguous full-day trading sessions case, except that the CEV exponent, $\gamma$, is set to be 1.0 (GARCH SV) and 1.5 .

The results for $\gamma=1.0$ and 1.5, together with those for the Heston SV case of $\gamma=.5$ investigated above, are summarized in Table 3. The biases and RMSEs for the two non-Heston cases are larger than the very small values for the Heston SV, but are nevertheless still small. This implies that $\mathrm{V}_{t, T}\left(\gamma+\frac{1}{2}\right) / \sqrt{\mathrm{V}_{t, T}(2 \gamma) \mathrm{V}_{t, T}} \approx 1$, at least under the parametric configurations that have been chosen here.

\section{Empirical results for intra-day high frequency S\&P 500 and VIX}

\footnotetext{
16 Jones (2003), Aït-Sahalia and Kimmel (2007), Duan and Yeh (2010), and Engle and Ishida (2002), among others, have reported empirical evidence pointing to $\gamma>0.5$ for equity returns data using the CEV SV and related models.
} 
As an empirical illustration ${ }^{17}$, we next consider applying the proposed estimators to the Heston SV model using intra-day tick data for the S\&P 500 index and VIX. The data for both series are obtained from TickData, and the sample period is from September 22, 2003 through to December 31, 2007 (giving 1,077 trading days). Based on a visual inspection of the volatility signature plots of the S\&P 500 and the VIX data in Figures 1 and 2, we choose five-minute intervals to calculate intra-day log differences of the S\&P 500 series and the differences in the VIX squared series to alleviate the effects of microstructure noise. The raw VIX data in annualized percentages are scaled to daily percentages and are squared before five-minute increments are taken.

The realized leverage obtained is -.5077 . The results of the joint GMM estimation of $(\kappa, \theta, \sigma)$ by $G M M-B Z 2$, and $(\kappa, \theta, \sigma, \rho)$ by $G M M-B Z-R L$, are summarized in Table 4. The standard errors are the usual asymptotic GMM standard errors. We need to be careful in interpreting the standard errors given to the $\rho$ estimates by $G M M-B Z-R L$ as we have not yet established the asymptotics for this estimator. It is likely that, in a double asymptotic framework, $T \rightarrow \infty$ and $\Delta t \rightarrow 0$, the $\rho$ component of the estimator GMM-BZ-RL is consistent for $\rho$ at a faster rate in the absence of measurement errors.

When we treat the data as arising from contiguous 24-hour sessions, $h=24$, the parameter of the long-run variance, $\theta$, is estimated to be .3647 by $G M M-B Z-R L$ and .3624 by $G M M-B Z 2$, which is not very different from the average RV, .4631 . When we treat the data as arising from non-contiguous 6.5-hour trading sessions, its estimates are much larger (namely, 1.339 by GMM-BZ-RL and 1.3388 by GMM-BZ2). The volatility-of-variance parameter, $\sigma$, is also estimated to be much larger under the correct $h=6.5$ assumption than under the incorrect $h=24$ assumption. These

\footnotetext{
${ }^{17}$ As a model for the processes of equity indices, the Heston SV specification has repeatedly been rejected in favour of more complicated models; see, for example, Andersen et al. (2002), Ait-Sahalia and Kimmel (2007), Jones (2003), Chernov et al. (2003), Pan (2002), Eraker et al. (2003), and Eraker (2004). Hence, we must go beyond estimating the Heston SV model for conducting a full-fledged empirical analysis.
} 
differences would translate to large differences in theoretical option prices.

\section{Conclusion}

We have proposed to use intraday high frequency model-free implied volatility data in constructing realized-measures-based moment conditions, in particular, cross-moment conditions, of the GMM/SMM estimator for continuous-time SV models of asset price processes. We have focused attention on Heston's affine-drift square-root SV model, and proposed the realized leverage as an estimator for the leverage parameter $\rho$, which is shown by simulation experiments to deliver accurate estimates of the leverage parameter under this model.

We also demonstrated by simulation experiments the importance of making proper adjustments to the moment conditions when realized measures are computed using data from non-contiguous non-full-day trading sessions. Analytical expressions for moment conditions are usually not available other than for the Heston SV specification, but the simulated method of moments (SMM) approach may be used. Our argument for the use of intraday implied volatility applies not only to the S\&P 500 index, but also to those equity price indices for which intraday high-frequency observations of a VIX-like model-free implied volatility are available. 


\section{References}

[1] Aït-Sahalia, Y., and R. Kimmel, 2007, Maximum likelihood estimation of stochastic volatility models, Journal of Financial Economics 83, 413-452.

[2] Andersen, T.G., L. Benzoni, and J. Lund, 2002, Estimating jump-diffusions for equity returns, Journal of Finance 57, 1239-84.

[3] Andersen, T.G., and T. Bollerslev, 1997, Intraday periodicity and volatility persistence in financial markets, Journal of Empirical Finance 4, 115-158.

[4] Bakshi, G., N. Ju, and H. Ou-Yang, 2006, Estimation of continuous-time models with an application to equity volatility dynamics, Journal of Financial Economics 82, 227-249.

[5] Barndorff-Nielsen, O.E., S.E. Graversen, J. Jacod, and N. Shephard, 2006, Limit theorems for bipower variation in financial econometrics, Econometric Theory 22, 677-719.

[6] Barndorff-Nielsen, O.E., and N. Shephard, 2004, Econometric analysis of realized covariation: High frequency based covariance, regression, and correlation in financial economics, Econometrica 72, 885-925.

[7] Black, F., 1976, Studies in stock price volatility changes, Proceedings of the 1976 Meetings of the American Statistical Association, Business and Economics Statistics, 177-181.

[8] Bollerslev, T., M. Gibson, and H. Zhou, 2011, Dynamic estimation of volatility risk premia and investor risk aversion from option-implied and realized volatilities, Journal of Econometrics 160, 235-245.

[9] Bollerslev, T., J. Litvinova, and G. Tauchen, 2006, Leverage and volatility feedback effects in high-frequency data, Journal of Financial Econometrics 4, 353-384.

[10] Bollerslev, T., and H. Zhou, 2002, Estimating stochastic volatility diffusion using conditional moments of integrated volatility, Journal of Econometrics 109, 33-65 (2004, Corrigendum, Journal of Econometrics 119, 221-222).

[11] Britten-Jones, M., and A. Neuberger, 2000, Option prices, implied price processes, and stochastic volatility, Journal of Finance 55, 839-866.

[12] CBOE, 2009, The CBOE volatility index - VIX, CBOE website. 
[13] Chernov, M., A.R. Gallant, E. Ghysels, and G. Tauchen, 2003, Alternative models for stock price dynamics, Journal of Econometrics 116, 225-257.

[14] Corradi, V., and W. Distaso, 2006, Semiparametric comparison of stochastic volatility models using realised measures, Review of Economic Studies 73, 635-667.

[15] Das, S.R., and R.K. Sundaram, 1999, Of smiles and smirks: A term structure perspective, Journal of Financial and Quantitative Analysis 34, 211-234.

[16] Demeterfi, K., E. Derman, M. Kamal and J. Zhou, 1999, More than you ever wanted to know about volatility swaps, Goldman Sachs Quantitative Strategies Research Notes.

[17] Dotsis, G., D. Psychoyios, and G. Skiadopoulos, 2007, An empirical comparison of continuous-time models of implied volatility indices, Journal of Banking and Finance 31, 3584-3603.

[18] Duan, J.-C., and C.-Y. Yeh, 2010, Jump and volatility risk premiums implied by VIX, Journal of Economic Dynamics \& Control 34, 2232-2244.

[19] Engle, R.F., and I. Ishida, 2002, Modeling variance of variance: The square-root, the affine, and the CEV GARCH models, Working Paper, New York University.

[20] Eraker, B., 2004, Do stock prices and volatility jump? Reconciling evidence from spot and option prices, Journal of Finance 59, 1367-1403.

[21] Eraker, B., M. Johannes, and N. Polson, 2003, The role of jumps in returns and volatility, Journal of Finance 58, 1269-1300.

[22] Fukasawa, M., I. Ishida, N. Maghrebi, K. Oya, M. Ubukata, and K. Yamazaki, 2010, Model-free implied volatility: From surface to index, forthcoming in International Journal of Theoretical and Applied Finance.

[23] Garcia, R., M.-A. Lewis, S. Pastorello, and É. Renault, 2011, Estimation of objective and risk-neutral distributions based on moments of integrated volatility, Journal of Econometrics 160, 22-32.

[24] Heston, S., 1993, A closed-form solution for options with stochastic volatility with applications to bond and currency options, Review of Financial Studies 6, 327-343.

[25] Jacod, J., and V. Todorov, 2010, Do price and volatility jump together, Annals of Applied Probability 20, 1425-1469.

[26] Jiang, G., and Y. Tian, 2005, The model-free implied volatility and its information content, Review of Financial Studies 18, 1305-1342. 
[27] Jones, C., 2003, The dynamics of stochastic volatility: Evidence from underlying and options markets, Journal of Econometrics 116, 181-224.

[28] Lee, S.S., and P.A. Mykland, 2008, Jumps in financial markets: A new nonparametric test and jump dynamics, Review of Financial Studies 21, 2535-2563.

[29] Nelson, D.B., 1990, ARCH models as diffusion approximation, Journal of Econometrics 45, 7-38.

[30] Pan, J., 2002, The jump-risk premia implicit in options: Evidence from an integrated time series, Journal of Financial Economics 63, 3-50.

[31] Rydberg, T.H., and N. Shephard, 2003, Dynamics of trade-by-trade price movements: Decomposition and models, Journal of Financial Econometrics 1, 2-25.

[32] Vortelinos, D.I., 2010, The properties of realized correlation: Evidence from the French, German and Greek equity markets, Quarterly Review of Economics and Finance 50, 273-290. 


\section{Appendix}

In this appendix, we derive the modifications (36) and (37) of the relations that lead to a set of conditional moment conditions for GMM estimation of the SV parameters to be applicable when each trading session lasts less than 24 hours $(\Delta<1)$ :

$$
\begin{gathered}
E\left[V_{t+\Delta} \mid \mathrm{H}_{t}\right]=\alpha_{\Delta} V_{t}+\beta_{\Delta}, \\
E\left[V_{t, t+\Delta} \mid \mathrm{H}_{t}\right]=a_{\Delta} V_{t}+b_{\Delta}, \\
\operatorname{Var}\left(V_{t, t+\Delta} \mid \mathrm{H}_{t}\right)=A_{\Delta} V_{t}+B_{\Delta}, \\
E\left[V_{t+\Delta}^{2} \mid \mathrm{H}_{t}\right]=\alpha_{\Delta}^{2} V_{t}^{2}+\left(C_{\Delta}+2 \alpha_{\Delta} \beta_{\Delta}\right) V_{t}+D_{\Delta}+\beta_{\Delta}^{2} .
\end{gathered}
$$

where $E\left[\square \mid \mathrm{H}_{t}\right]$ is the expectation under the physical measure, conditional on

$\mathrm{H}_{t}:=\sigma\left\{V_{s}: s \leq t\right\}$. These are, respectively, equations (A.1), (A.2), (A.5) and (A.6) of BZ, which lead to:

$$
\begin{aligned}
& E\left[\mathrm{~V}_{t+1, t+1+\Delta} \mid \mathrm{H}_{t}\right]=E\left[E\left[\mathrm{~V}_{t+1, t+1+\Delta} \mid \mathrm{H}_{t+1}\right] \mid \mathrm{H}_{t}\right] \\
& \quad=a_{\Delta} \alpha_{1} V_{t}+a_{\Delta} \beta_{1}+b_{\Delta}=\alpha_{1} E\left[\mathrm{~V}_{t, t+\Delta} \mid \mathrm{H}_{t}\right]+\beta_{1} \Delta,
\end{aligned}
$$

Since $\mathrm{G} \subset \mathrm{H}_{t}$, we obtain, by the law of iterated expectations,

$$
E_{t}^{\Delta}\left[\mathrm{V}_{t+1, t+1+\Delta}\right]=\alpha_{1} E_{t}^{\Delta}\left[\mathrm{V}_{t, t+\Delta}\right]+\beta_{1} \Delta
$$

which reduces to equation (6) of BZ if $\Delta=1$. Furthermore, we have:

$$
\begin{aligned}
E\left[\mathrm{~V}_{t, t+\Delta}^{2}\right. & \left.\mid \mathrm{H}_{t}\right]=\operatorname{Var}\left(\mathrm{V}_{t, t+\Delta} \mid \mathrm{H}_{t}\right)+\left(E\left[\mathrm{~V}_{t, t+\Delta} \mid \mathrm{H}_{t}\right]\right)^{2} \\
& =A_{\Delta} V_{t}+B_{\Delta}+\left(a_{\Delta} V_{t}+b_{\Delta}\right)^{2} \\
& =A_{\Delta} V_{t}+B_{\Delta}+a_{\Delta}^{2} V_{t}^{2}+b_{\Delta}^{2}+2 a_{\Delta} b_{\Delta} V_{t} \\
& =a_{\Delta}^{2} V_{t}^{2}+\left(A_{\Delta}+2 a_{\Delta} b_{\Delta}\right) V_{t}+B_{\Delta}+b_{\Delta}^{2}
\end{aligned}
$$


which is essentially equation (A.7) in BZ, and

$$
\begin{gathered}
E\left[\mathrm{~V}_{t+1, t+1+\Delta}^{2} \mid \mathrm{H}_{t}\right]=E\left[E\left[\mathrm{~V}_{t+1, t+1+\Delta}^{2} \mid \mathrm{H}_{t+1}\right] \mid \mathrm{H}_{t}\right] \\
=E\left[a_{\Delta}^{2} V_{t+1}^{2}+\left(A_{\Delta}+2 a_{\Delta} b_{\Delta}\right) V_{t+1}+B_{\Delta}+b_{\Delta}^{2} \mid \mathrm{H}_{t}\right] \\
=a_{\Delta}^{2}\left(\alpha_{1}^{2} V_{t}^{2}+\left(C_{1}+2 \alpha_{1} \beta_{1}\right) V_{t}+D_{1}+\beta_{1}^{2}\right)+\left(A_{\Delta}+2 a_{\Delta} b_{\Delta}\right)\left(\alpha_{1} V_{t}+\beta_{1}\right)+B_{\Delta}+b_{\Delta}^{2} \\
=\alpha_{1}^{2} E\left[\mathrm{~V}_{t, t+\Delta}^{2} \mid \mathrm{H}_{t}\right]+\left[a_{\Delta}^{2}\left(C_{1}+2 \alpha_{1} \beta_{1}\right)+\left(\alpha_{1}-\alpha_{1}^{2}\right)\left(A_{\Delta}+2 a_{\Delta} b_{\Delta}\right)\right] V_{t} \\
+\left(A_{\Delta}+2 a_{\Delta} b_{\Delta}\right) \beta_{1}+a_{\Delta}^{2}\left(D_{1}+\beta_{1}^{2}\right)+\left(1-\alpha_{1}^{2}\right)\left(B_{\Delta}+b_{\Delta}^{2}\right) \\
=\alpha_{1}^{2} E\left[\mathrm{~V}_{t, t+\Delta}^{2} \mid \mathrm{H}_{t}\right]+\left[a_{\Delta}\left(C_{1}+2 \alpha_{1} \beta_{1}\right)+\left(\alpha_{1}-\alpha_{1}^{2}\right)\left(a_{\Delta}^{-1} A_{\Delta}+2 b_{\Delta}\right)\right] E\left[\mathrm{~V}_{t, t+\Delta} \mid \mathrm{H}_{t}\right] \\
-\left[a_{\Delta}\left(C_{1}+2 \alpha_{1} \beta_{1}\right)+\left(\alpha_{1}-\alpha_{1}^{2}\right)\left(a_{\Delta}^{-1} A_{\Delta}+2 b_{\Delta}\right)\right] b_{\Delta} \\
+\left(A_{\Delta}+2 a_{\Delta} b_{\Delta}\right) \beta_{1}+a_{\Delta}^{2}\left(D_{1}+\beta_{1}^{2}\right)+\left(1-\alpha_{1}^{2}\right)\left(B_{\Delta}+b_{\Delta}^{2}\right) \\
=\alpha_{1}^{2} E\left[\mathrm{~V}_{t, t+\Delta}^{2} \mid \mathrm{H}_{t}\right]+I_{\Delta} E\left[\mathrm{~V}_{t, t+\Delta} \mid \mathrm{H}_{t}\right]+J_{\Delta},
\end{gathered}
$$

By the law of iterated expectations, we obtain:

$$
E_{t}^{\Delta}\left[\mathrm{V}_{t+1, t+1+\Delta}^{2}\right]=\alpha_{1}^{2} E_{t}^{\Delta}\left[\mathrm{V}_{t, t+\Delta}^{2}\right]+I_{\Delta} E_{t}^{\Delta}\left[\mathrm{V}_{t, t+\Delta}\right]+J_{\Delta},
$$

which reduces to equation (10) in BZ if $\Delta=1$. 
Figure 1: Volatility Signature Plot (S\&P 500 Index)

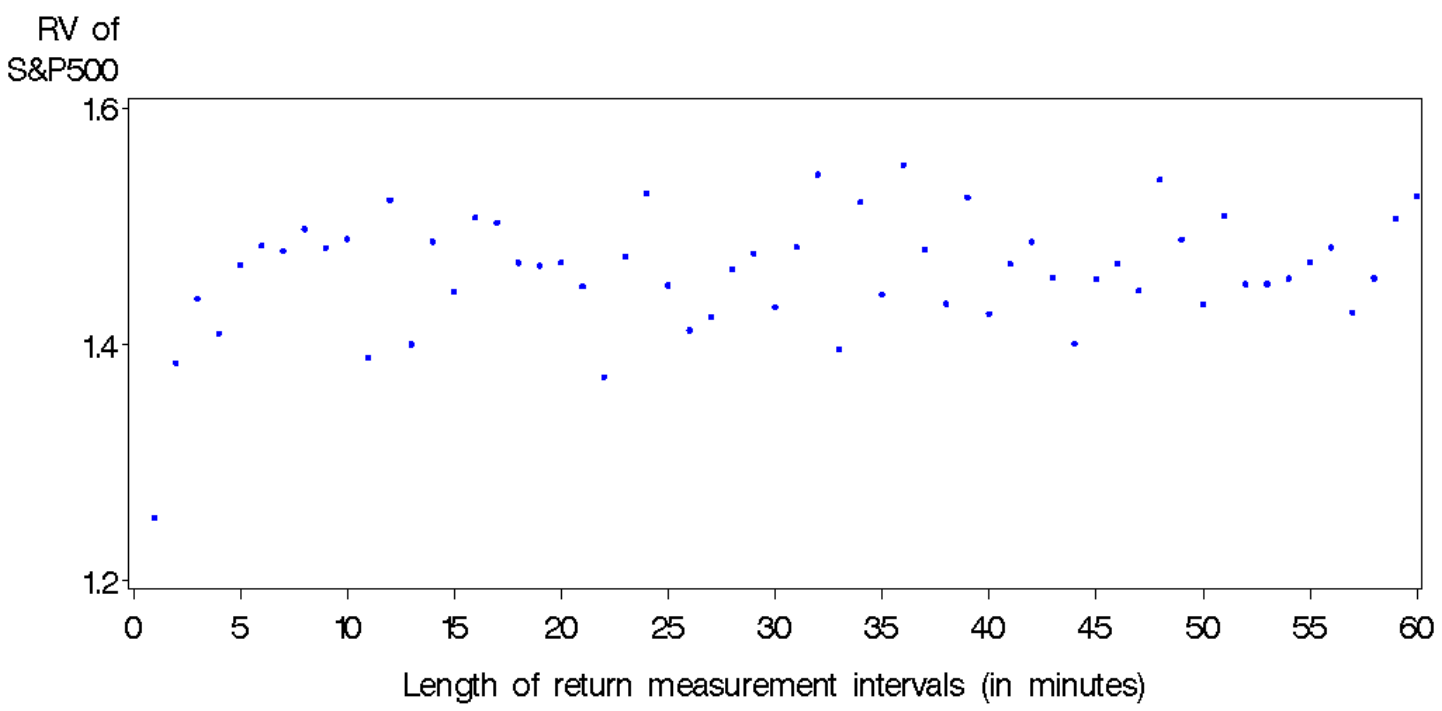

Figure 2: Volatility Signature Plot (Squared VIX)

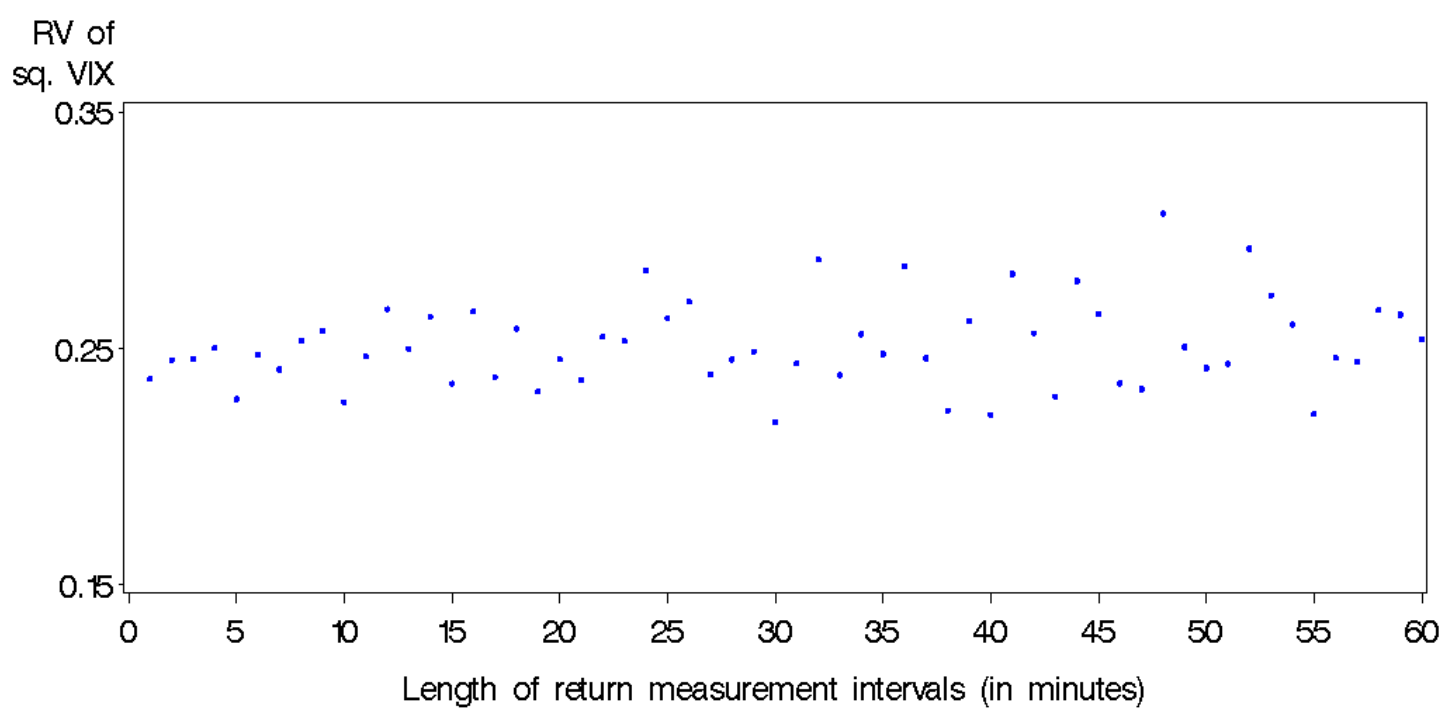


Table 1

\section{Monte Carlo Experiment Results}

\section{Panel A}

True Parameter Set 1: $\kappa=.1, \quad \theta=.25, \quad \sigma=.1, \quad \rho=-.5$

(faster mean reversion)

\begin{tabular}{|c|c|c|c|c|c|c|c|c|}
\hline & \multicolumn{4}{|c|}{ Bias $\times 100$} & \multicolumn{4}{|c|}{ RMSE $\times 100$} \\
\hline & $\kappa$ & $\theta$ & $\sigma$ & $\rho$ & $\kappa$ & $\theta$ & $\sigma$ & $\rho$ \\
\hline$G M M-B Z 1$ & .1116 & .5777 & -.0146 & 28.5206 & 1.8218 & 39.8720 & .6407 & 45.8699 \\
\hline$G M M-B Z-R L$ & .1720 & -.3069 & .0011 & .0715 & 1.7677 & 2.4971 & .5380 & .1612 \\
\hline$R L$ & & & & .0031 & & & & .1565 \\
\hline$G M M-B Z 2$ & .1592 & -.2995 & .0041 & & 1.7647 & 1.7236 & .5359 & \\
\hline
\end{tabular}

\section{Panel B}

True Parameter Set 2: $\kappa=.05, \theta=.25, \quad \sigma=.1, \quad \rho=-.5$

(slower mean reversion)

\begin{tabular}{|c|c|c|c|c|c|c|c|c|}
\hline & \multicolumn{4}{|c|}{ Bias $\times 100$} & \multicolumn{4}{|c|}{$\mathrm{RMSE} \times 100$} \\
\hline & $\kappa$ & $\theta$ & $\sigma$ & $\rho$ & $\kappa$ & $\theta$ & $\sigma$ & $\rho$ \\
\hline$G M M-B Z 1$ & .3399 & -.0464 & -.0889 & 28.7774 & 1.3005 & 21.5115 & .6402 & 46.8173 \\
\hline$G M M-B Z-R L$ & .3940 & -.6700 & -.0792 & .0702 & 1.2910 & 3.4574 & .5673 & .1601 \\
\hline$R L$ & & & & .0001 & & & & .1666 \\
\hline$G M M-B Z 2$ & .3819 & -.6392 & -.0736 & & 1.2869 & 3.4551 & .5469 & \\
\hline
\end{tabular}


Table 2

Effects of Not Properly Adjusting the Moments for Market Closure

\begin{tabular}{|c|c|c|c|c|c|c|c|c|}
\hline \multirow{3}{*}{ Hours } & \multicolumn{4}{|c|}{ Bias $\times 100$} & \multicolumn{4}{|c|}{$\mathrm{RMSE} \times 100$} \\
\hline & \multicolumn{3}{|c|}{$B Z$} & \multirow{2}{*}{$\begin{array}{c}R L \\
\rho\end{array}$} & \multicolumn{3}{|c|}{$\mathrm{BZ}$} & \multirow{2}{*}{$\frac{R L}{\rho}$} \\
\hline & $\kappa$ & $\theta$ & $\sigma$ & & $\kappa$ & $\theta$ & $\sigma$ & \\
\hline 6 & .1358 & -.1080 & .5870 & .0019 & 2.0951 & 1.6858 & 1.0365 & .3079 \\
\hline 12 & .1202 & -12.5446 & -2.4815 & & 2.0881 & 12.5725 & 2.5544 & \\
\hline 18 & .0993 & -16.6894 & -3.8365 & & 2.0861 & 16.6987 & 3.8689 & \\
\hline 24 & .0839 & -18.7617 & -4.6396 & & 2.0846 & 18.7664 & 4.6603 & \\
\hline
\end{tabular}


Table 3

Realized Leverage as an Estimator of $\rho$ under Heston $(\gamma=.5)$ and Non-Heston CEV $(\gamma=1.0, \gamma=1.5)$

\begin{tabular}{ccc}
\hline$\gamma$ & Bias $\times 100$ & RMSE $\times 100$ \\
\hline .5 & .0031 & .1565 \\
1.0 & .3193 & .3478 \\
1.5 & .3285 & .3554 \\
\hline
\end{tabular}


Table 4

GMM Estimation Using High-frequency S\&P 500 and VIX

\begin{tabular}{|c|c|c|c|c|c|c|c|c|}
\hline & \multicolumn{4}{|c|}{$h=24$} & \multicolumn{4}{|c|}{$h=6.5$} \\
\hline & $\kappa$ & $\theta$ & $\sigma$ & $\rho$ & $\kappa$ & $\theta$ & $\sigma$ & $\rho$ \\
\hline$G M M-B Z-R L$ & .1584 & .3647 & .2457 & -.5736 & .1581 & 1.3390 & .4818 & -.5734 \\
\hline (S.E.) & (.0709) & $(.0687)$ & $(.1734)$ & $(.0095)$ & $(.0722)$ & $(.2424)$ & $(.3205)$ & $(.0095)$ \\
\hline$G M M-B Z 2$ & .1576 & .3624 & .2438 & & .1589 & 1.3388 & .4917 & \\
\hline (S.E.) & $(.0708)$ & $(.0692)$ & $(.1746)$ & & $(.0723)$ & $(.2413)$ & $(.3147)$ & \\
\hline
\end{tabular}

